Conclusion: The determination of ORM1 and HPT in sera provides novel information useful for patient stratification, which might improve diagnostic and prognostic approaches and facilitate the development of personalized medicine strategies in RA.

Acknowledgements: This work is supported by grants from Fondo de Investigación Sanitaria (RD16/0012/0002, PT17/0019/0014) integrated in the National Plan for Scientific Program, Development and Technological Innovation 20132016 and funded by the ISCIII-General Subdirection of Assessment and Promotion of Research-European Regional Development Fund (FEDER) "A way of making Europe".

Disclosure of Interests: None declared

DOI: 10.1136/annrheumdis-2021-eular.2533

\section{POS0186 \\ METABOLOMIC SIGNATURES FOR KNEE CARTILAGE VOLUME LOSS OVER 10 YEARS}

Z. Xie $^{1,2}$, D. Aitken ${ }^{3}$, M. Liu' ${ }^{1}$, G. Lei ${ }^{2}$, G. Jones ${ }^{3}$, G. Zhai ${ }^{1}{ }^{1}$ Memorial University of Newfoundland, Division of Biomedical Sciences (Genetics), Faculty of Medicine, St. John's, Canada; ${ }^{2}$ Central South University, Xiangya Hospital, Changsha, China; ${ }^{3}$ University of Tasmania, Menzies Institute for Medical Research, Hobart, Australia

Background: Osteoarthritis $(\mathrm{OA})$ is the most common form of arthritis, and its impact is increasing year by year due to an aging population and lack of effective treatments. One of the main structural pathological changes of $O A$ is the loss of articular cartilage. Tools that can predict cartilage loss would help identify people at high risk, thus preventing OA development.

Objectives: Using a metabolomics approach, the current study aimed to identify serum metabolomic signatures for predicting the loss of knee cartilage volume over 10 years in a well-established community-based cohort - the Tasmania Older Adult Cohort (TASOAC).

Methods: TASOAC is an on-going, prospective, population-based study of older adults who were randomly selected from the roll of electors in Southern Tasmania, Australia. Participants had a right knee magnetic resonance imaging (MRI) scan at baseline and a 10-year follow-up. Cartilage volume was measured in the medial, lateral, and patellar compartments and change in cartilage volume over 10 years was calculated as percentage change per year. Fasting serum samples collected at 2.6-year follow-up were metabolomically profiled using the TMIC Prime Metabolomics Profiling Assay which measures a total of 143 metabolites. 129 metabolite concentrations passed the quality control and the pairwise ratios of them as the proxies of enzymatic reaction were calculated. Linear regression models were used to test the association between each of the metabolite ratios and change in cartilage volume in each of the knee compartments with adjustment for age, sex, and body mass index (BMI). The significance was defined at $a=3.0 \times 10^{-6}$ to control multiple testing of 16,512 ratios with Bonferroni method.

Results: A total of 344 participants ( $51 \%$ females) were included. The mean baseline age was $62.83 \pm 6.13$ years and the mean BMI was $27.48 \pm 4.41 \mathrm{~kg} / \mathrm{m}^{2}$. The average follow-up time was $10.84 \pm 0.66$ years. Cartilage volume reduced by $1.34 \pm 0.72 \%, 1.06 \pm 0.58 \%$, and $0.98 \pm 0.46 \%$ per year in the medial, lateral, and patellar compartments, respectively. Our data showed that an increased ratio of hexadecenoylcarnitine (C16:1) to tetradecanoylcarnitine $(\mathrm{C} 14)$ was associated with a $0.12 \pm 0.02 \%$ per year reduction in patellar cartilage volume $\left(p=8.80 \times 10^{-7}\right)$. An increased ratio of hexadecenoylcarnitine (C16:1) to dodecanoylcarnitine (C12) was also associated with a $0.12 \pm 0.02 \%$ per year reduction in patellar cartilage volume $\left(p=2.66 \times 10^{-6}\right)$. While there were several metabolite ratios associated with cartilage volume loss in the medial and lateral compartments, none of them reached the predefined significance level.

Conclusion: Our data suggested that alteration of fatty acid $\beta$-oxidation is involved in knee cartilage loss, especially in the patellar compartment, and the serum ratio of $\mathrm{C} 16: 1$ to $\mathrm{C} 14$ and to $\mathrm{C} 12$ could be used to predict long-term patellar cartilage loss.

Acknowledgements: We thank all the study participants who made the study possible. The original TASOAC study was supported by the National Health and Medical Research Council (NHMRC) and the current study was supported by the Canadian Institutes of Health Research (ClHR).

Disclosure of Interests: None declared

DOI: 10.1136/annrheumdis-2021-eular.2735

\section{POS0187 THE PROGNOSTIC FACTORS IN PREDICTING RELAPSE FOR IGG4-RELATED DISEASE: A LONG-TERM STUDY BASED ON THE CLINICAL AND PATHOLOGICAL CHARACTERISTICS OF THE PATIENTS}

Z. Ji ${ }^{1}$, L. Chen ${ }^{2}$, L. Ma ${ }^{1}$, L. Zhang ${ }^{1}$, H. Chen ${ }^{1}$, L. Ma ${ }^{1}$, L. Jiang ${ }^{1} .{ }^{1}$ Shanghai Zhongshan hospital, Fudan University, Rheumatology, Shanghai, China; ${ }^{2}$ Shanghai Zhongshan hospital, Fudan University, Pathology, Shanghai, China
Background: The relationship between the pathological findings and disease relapse has not been well established.

Objectives: We aim to investigate the clinical and pathological manifestations in relation with disease relapse in IgG4-RD, as well as identify prognostic factors in predicting relapsed disease.

Methods: This study enrolled 71 patients newly diagnosed with IgG4-RD between Jan 2011 and April 2020, all of whom had received pathological examinations. Their pathological manifestations and clinical data were collected. Multivariate Cox regression and AUC (area under curve) were used to identify predictors for relapsed disease and assess the predictive value of these predictors, respectively. Results: During a follow-up period of 26 (range, 6-123) months, 4.2\% (3/71) patients died. The remaining 68 patients were all treated with glucocorticoids with or without immunosuppressor continuously. By the end of follow-up, 47 (69.1\%) patients sustained clinical remission, and 21 (30.9\%) patients suffered relapsed disease with a median relapse time at 10 (6-30) months. We found that IgG4 $\geq$ 6.5g/L (OR 1.52-11.06), IgG $\geq 20.8 \mathrm{~g} / \mathrm{L}$ (OR 1.11-7.23), IgG4-RD responder index $(\mathrm{RI}) \geq 9$ (OR 1.28-11.37), and more IgG4 ${ }^{+}$plasma cell infiltration ( $\geq 60 / \mathrm{HPF}$ in visceral organs, or $\geq 200$ / HPF in head and neck organs) (OR 1.79-22.41) were all independent predictive factors for disease relapse. A prognostic score was explored for predicting recurrence in IgG4-RD, including three predictive factors (IgG $\geq 20.8 \mathrm{~g} / \mathrm{L}, \lg \mathrm{G} 4-\mathrm{RD} \mathrm{RI} \geq 9$, and more $\operatorname{lgG} 4^{+}$plasma cell infiltration). The three-year relapse rate for the patients with no, one, two, and three risk factors were $0 \%, 27.3 \%, 66.7 \%$, and $100 \%$, respectively.

Conclusion: Patients' earlier lgG4 $\geq 6.5 \mathrm{~g} / \mathrm{L}, \mathrm{IgG} \geq 20.8 \mathrm{~g} / \mathrm{L}$, IgG4-RD RI $\geq 9$, and more $\operatorname{lgG} 4^{+}$plasma cell infiltration independently predicted disease relapse. We explored a prognostic score for predicting recurrence in IgG4-RD include three predictive factors ( $\operatorname{lgG} \geq 20.8 \mathrm{~g} / \mathrm{L}$, IgG4-RD $\mathrm{RI} \geq 9$, and more $\operatorname{lgG} 4^{+}$plasma cell infiltration), which might be used to evaluate the risk of recurrence in IgG4-RD.

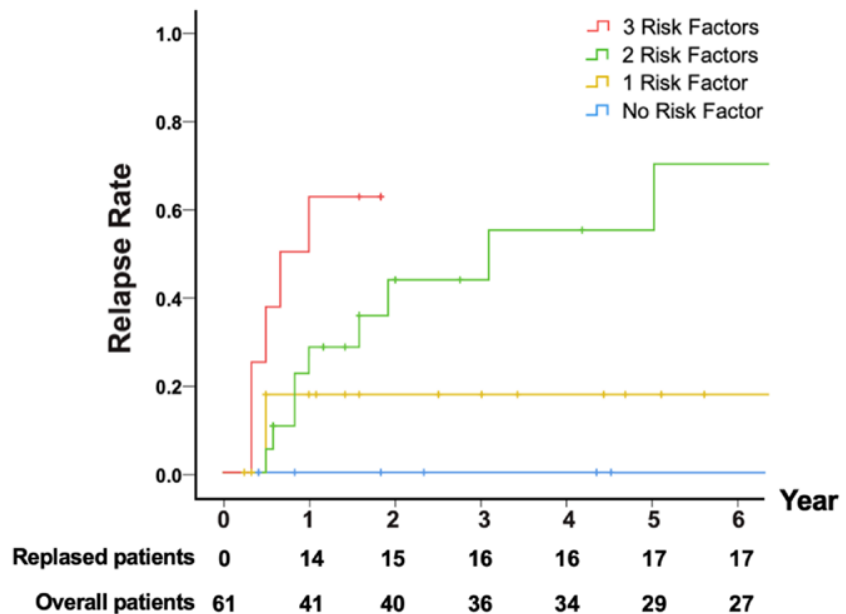

\section{REFERENCES:}

[1] DESHPANDE V, ZEN Y, CHAN J K, et al. Consensus statement on the pathology of IgG4-related disease[J]. Mod Pathol,2012,25(9): 1181-1192.

[2] JENNETTE J C, FALK R J, BACON P A, et al. 2012 revised International Chapel Hill Consensus Conference Nomenclature of Vasculitides[J]. Arthritis Rheum,2013,65(1): 1-11.

[3] OKAZAKI K, UMEHARA H. Are Classification Criteria for IgG4-RD Now Pos sible? The Concept of IgG4-Related Disease and Proposal of Comprehensive Diagnostic Criteria in Japan[J]. Int J Rheumatol,2012,2012: 357071.

[4] SHIMOSEGAWA T, CHARI S T, FRULLONI L, et al. International consensus diagnostic criteria for autoimmune pancreatitis: guidelines of the International Association of Pancreatology[J]. Pancreas,2011,40(3): 352-358.

[5] OHARA H, OKAZAKI K, TSUBOUCHI H, et al. Clinical diagnostic criteria of IgG4-related sclerosing cholangitis 2012[J]. J Hepatobiliary Pancreat Sci,2012,19(5): 536-542.

[6] KAWANO M, SAEKI T, NAKASHIMA H, et al. Proposal for diagnostic criteria for IgG4-related kidney disease[J]. Clin Exp Nephrol,2011,15(5): 615-626.

[7] GOTO H, TAKAHIRA M, AZUMI A. Diagnostic criteria for IgG4-related ophthalmic disease[J]. Jpn J Ophthalmol,2015,59(1): 1-7.

[8] MATSUI S, YAMAMOTO H, MINAMOTO S, et al. Proposed diagnostic criteria for IgG4-related respiratory disease[J]. Respir Investig,2016,54(2): 130-132.

[9] WEN ZHANG J H S. Management of IgG4-related disease[J]. Lancet Rheumatol,2019,1: e55-e65.

[10] EBBO M, DANIEL L, PAVIC M, et al. IgG4-related systemic disease: features and treatment response in a French cohort: results of a multicenter regis try[J]. Medicine (Baltimore),2012,91(1): 49-56.

Disclosure of Interests: None declared

DOI: 10.1136/annrheumdis-2021-eular.3391 